\title{
Influence of Shear Bonding Strength of Lithium Disilicate to Enamel under Different Surface Treatments
}

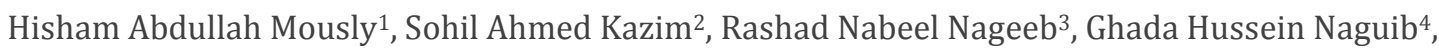 \\ Mohamed Tharwat Hamed ${ }^{5}$
}

${ }^{1}$ Department of Oral \& Maxillofacial Prosthodontics, King Abdulaziz University, Jeddah, Saudi Arabia. ${ }^{2}$ King Abdulaziz University, Jeddah, Saudi Arabia. ${ }^{3}$ King Abdulaziz University, Jeddah, Saudi Arabia. ${ }^{4}$ Department of Restorative Dentistry, King Abdulaziz University, Jeddah, Saudi Arabia, and the Department of Oral Biology, Cairo University, Cairo, Egypt. ${ }^{5}$ Department of Oral \& Maxillofacial Prosthodontics, King Abdulaziz University, Jeddah, Saudi Arabia and the Department of Fixed Prosthodontics, Cairo University Faculty of Dentistry, Cairo, Egypt.

\section{ABSTRACT}

\section{BACKGROUND}

Accurate diagnosis and proper treatment planning should be established before making any prosthetic rehabilitation to restore the good appearance of the tooth, a better smile, phonetics, and to achieve perfect occlusion/mastication. We wanted to evaluate the changes of surface treatment protocols on the tensile bonding strength between enamel and ceramic restorations (lithium disilicate).

\section{METHODS}

A total of 20 freshly extracted, non-carious teeth were stored in normal saline solution at $25^{\circ} \mathrm{C}$ until used. Clean enamel surfaces $2 \mathrm{~mm} \mathrm{X} 2 \mathrm{~mm}$ were obtained from the extracted teeth by using wheel diamond bur. Each enamel side received one Emax sprue, so four sprues of different surface treatment protocols per tooth (G1 TS: Etching, bonding, and curing. CS: Etching, monobond, curing, overall light cure) (G2 TS: Bonding, curing, self-etch / self-adhesive resin cement, overall light cure. CS: Monobond etch and prime) (G3 TS: Etching, bonding without curing. CS: Etching, monobond, curing, overall light cure) (G4 TS: Etching, bonding, curing. CS: Etching, monobond, bonding, curing, light-cure resin cement, overall light cure). Tensile bonding strength was measured using the Instron testing machine. One-way ANOVA test was used to analyse the data.

\section{RESULTS}

The highest mean was observed in Group 3 (124.34 \pm 43.47$)$ followed by Group 1 (104.29 \pm 50.09$)$, which is control group. In contrast, the lowest mean was observed in Group 2 (83.64 \pm 53.56$)$ and Group 4 (94.14 \pm 57.91$)$. The results of ANOVA test have shown a significant difference between the surface treatment groups at $5 \%$ significance level.

\section{CONCLUSIONS}

Hydrofluoric acid and primer/silane coupling agent create a porous surface on the ceramic that allow a good interaction with silane coupling agent.

\section{KEY WORDS}

Enamel, Hydrofluoric Acid, Lithium Disilicate, Primer, Silane, Tensile Bonding Strength
Corresponding Author:

Mohamed Tharwat Hamed, BDS, MSD, CAGS, MPH, MPHE, DMSC. Department of Fixed Prosthodontics, Cairo University School of Dentistry, Alkasr Aleiny, Almanyal, Cairo, Egypt. E-mail: mohsarwat62@gmail.com

DOI: $10.14260 / j e m d s / 2020 / 154$

Financial or Other Competing Interests: None.

How to Cite This Article:

Mously HA, Kazim SA, Nageeb RN, et al. Influence of shear bonding strength of lithium disilicate to enamel under different surface treatments. J. Evolution Med. Dent. Sci.2020;9(10):705-710, 10.14260/jemds/2020/154

Submission 04-10-2019,

Peer Review 07-02-2020,

Acceptance 14-02-2020,

Published 09-02-2020. 


\section{BACKGROUND}

The success and reliability of ceramics bonding to dental substrate result in the long-term survival of aesthetic restorations. Several surface treatments regarding chemical bonding and micromechanical retention were suggested to enhance resin cement bonding to ceramics.1,2 The best surface treatment to be utilized is determined through the composition of the ceramic. Similarly, the effect of etching protocols used on glass matrix ceramics has been examined by several studies. ${ }^{3-6}$ In this regard, acid etching is considered as the most effective treatment method for the bonding surface of glass ceramic restorations. Selective removal of the glassy matrix of silicate ceramics allows micromechanical interlocking of the luting composite, resulting in a micromorphological 3D porous surface. ${ }^{4}$ The acid type and its concentration, the treated ceramic type, and the etching time are dependent components for achieving successful effects of acid etching. ${ }^{5}$

One of the most often used acids is the hydrofluoric acid while the ammonium hydrogen difluoride and acidulated phosphate fluoride is also frequently observed. ${ }^{6}$ Some silicon ammonium fluoride and tetrafluoride are created due to the mixture of silica matrix and the ammonium hydrogen difluoride. The use of this acid is examined as an intermediate or a glass etching for the hydrofluoric acid production. ${ }^{7}$ higher bond strengths are generated through hydrofluoric acid etching followed by silanization as compared to the treatment alone. The application of silanization is understood in creating covalent bonding and hydrogen bonding between the ceramic and the resin as well as escalated wettability of the ceramic surface whereas the mechanical interlocking is provided by etching. ${ }^{8}$ Silane promotes the chemical adhesion to promote higher mean bond strength values as compared to the micromechanical retention created by any etchant.

Recently, the instigation of a simplified acid ceramic primer has claimed to perform a mild acid silanization and etching using a single solution. ${ }^{9}$ This monobond Etch \& Prime (MEP) and one bottle system combine silane and ammonium polyfluoride on the basis of trimethoxy propyl methacrylate to show a thin chemically bonded layer. ${ }^{10}$ The introduction of this primer was to simplify the bonding procedure by priming glass and etching ceramics using a one-step process. In particular, a milder acidity is observed in ammonium polyfluoride as compared to hydrofluoric acid, resulting in weaker etching pattern. ${ }^{11}$ Several prior studies have been conducted to compare the efficiency of the protocol using 2step surface treatments and silane followed by concentration and application times. ${ }^{12-14}$ Comparative outcomes are provided by these studies on field-emission scanning electron microscopic analysis, micromorphological analysis, tensile bond strength, shear bond strength, and contact angle.

The bonding strength is the main source of the Emax/lithium disilicate restorations to compare the other types of crowns, which include Zirconia or PFM, which retains the cement itself for producing the E-max unique. ${ }^{15}$ Not only the preparation designs, resistance, and retention forms have been reported as highest factors that affect the longevity of the bonding strength. However, there are many other factors considered for the type of restorations on tooth surface or its natural teeth with no restorations on it. ${ }^{16,17}$ Individuals are looking for tooth coloured and aesthetic crowns specifically in the anterior region, which reduce all ceramic restorations. ${ }^{18}$ Furthermore, the reliance of a durable ceramic crown is on the cementation technique and the cement type. Ceramic restorations are recommended for different types of cement, if a lithium disilicate restoration is used based on the silica. ${ }^{19}$

The resin cements have many advantages such as it comes with different shades and highly aesthetic materials. Most importantly, the bond strength between the ceramic/lithium disilicate and tooth structure is considered due to limited literatures. ${ }^{14}$ Also, the surface treatment of the fitting side of the ceramic restorations should take place to increase the bond strength. The usual technique and materials to increase the bonding strength are divided into tooth part and ceramic part surface treatment. The conventional surface treatment onto the tooth side is to etch by $35 \%$ phosphoric acid and then resin bond. Hydrofluoric acid and primer/silane coupling agents were used for the ceramic side as they create a porous surface on the ceramic that allow a good interaction with silane coupling agent. ${ }^{11}$ Despite of the advantages of the HF acid etching followed by silane application, the protocol of etching ceramics is still not clear. ${ }^{10}$ In this regard, the study aims to evaluate the changes in surface treatment protocols on the tensile bonding strength between enamel and ceramic restorations (lithium disilicate). Following hypotheses have been proposed based on the aforementioned objective.

\section{Hypothesis}

Ho: $_{0}$ There is no difference in tensile bonding strength between surface treatment protocols.

$\mathbf{H}_{1}$ : There will be a difference in tensile bonding strength between surface treatment protocols

\section{METHODS}

\section{Experimental Design and Specimen Preparation}

A total of 20 freshly extracted, non-carious molars teeth were stored in normal saline solution at $25^{\circ} \mathrm{C}$ until used. Every tooth was mounted in orthoresins block [Protechno Famadent, S. L. U. 17469 Vilamalla (Girona) Spain]. The enamel of the four tooth surfaces (buccal, lingual, mesial, and distal) were roughened by using Wheel Diamond Bur which represented the tooth bonding surface. 80 heat pressed E-Max sprues (Ivoclar Vivadent, Schaan, Liechtenstein) of clean ceramic were used in this study. Each ceramic sprue had $2 \times 2$ surface diameter. Then, those E-max sprues were cemented on the different surface sides of the same tooth which carried different surface treatments protocols. All sides of toothceramic surfaces had the same bonding surface area.

\section{Microshear Bond Strength ( $\mu S B S$ )}

The following experimental groups were formed for determining microshear bond strength test.

\section{Group 1 (Control)}

On the tooth surface

1. $37 \%$ Phosphoric Acid semi Gel Meta Etchant (meta Biomed CO. LTD, United Kingdom).

2. Washing for 10 seconds then drying for 10 seconds. 
3. Bonding with resin bonding agent (Optibond, Kerr via Passanti, Italia).

4. LED Light cure 10 seconds.

On the ceramic surface

1. Etch with $9 \%$ HF acid (Ultradent Porcelain Etch $9 \%$ buffered hydrofluoric acid) for 20 seconds.

2. Washing for 10 seconds then drying for 10 seconds.

3. Monobond N Refill (Ivoclar Vivadent AG FL-9494, Schaan/Liechtenstein) for 60 second. Then gentle air drying for 10 seconds.

4. Light-cure resin cement (Kerr corporation NX3 NEXUS Third Generation, U. S. A).

5. Overall LED Light cure for 30 seconds.

\section{Group 2}

On the tooth surface

1. Bonding with resin bonding agent (Optibond, Kerr via Passanti, Italia).

2. LED Light cure 10 seconds.

3. Self-Etch / Self-Adhesive Resin Cement (Maxcem Elite Clear, Kerr via Passanti, Italia).

4. Overall LED Light cure for 30 seconds.

On the ceramic surface

1. Monobond Etch and prime (Ivoclar Vivadent AG 9494, Schaan/Liechtenstein) for 60 seconds.

2. Washing for 10 seconds then drying for 10 seconds.

\section{Group 3}

On the tooth surface

1-37\% Phosphoric Acid semi Gel Meta Etchant (Meta Biomed CO.LTD, United Kingdom)

2- Washing for 10 seconds then drying for 10 seconds

3- Bonding with resin bonding agent (Optibond, Kerr via Passanti, Italia) without curing

On the ceramic surface

1. Etch with $9 \%$ HF acid (Ultradent Porcelain Etch $9 \%$ buffered hydrofluoric acid) for 20 seconds.

2. Washing for 10 seconds then drying for 10 seconds.

3. Monobond N Refill (Ivoclar Vivadent AG FL-9494, Schaan/Liechtenstein) for 60 second. Then gentle air drying for 10 seconds.

4. Light-cure resin cement (Kerr corporation NX3 NEXUS Third Generation, U.S.A).

5. Overall LED Light cure for 30 seconds.

\section{Group 4}

On the tooth surface

1. $37 \%$ Phosphoric Acid semi Gel Meta Etchant (Meta Biomed Co. Ltd., United Kingdom).

2. Washing for 10 seconds then drying for 10 seconds

3. Bonding with resin bonding agent (Optibond, Kerr via Passanti, Italia).

4. LED Light cure 10 seconds.

On the ceramic surface

1. Etch with $9 \% \mathrm{HF}$ acid (Ultradent Porcelain Etch $9 \%$ buffered hydrofluoric acid) for 20 seconds.

2. Washing for 10 seconds then drying for 10 seconds.
3. Monobond N Refill (Ivoclar Vivadent AG FL-9494, Schaan/Liechtenstein) for 60 seconds. Then gentle air drying for 10 seconds.

4. Bonding with resin bonding agent (Optibond, Kerr via Passanti, Italia)

5. LED Light cure 10 seconds.

6. Light-cure resin cement (Kerr corporation NX3 NEXUS Third Generation, U. S. A).

7. Overall LED Light cure for 30 seconds.

The specimens were mounted on the bottom fixture of a universal testing machine (the Instron testing machine) and the top apparatus grips the bonding resin cement.

\section{Formula for the Calculation of Tensile Bond Strengths $\sigma=\mathrm{P} / \mathrm{A}$}

where,

$\sigma$ is the tensile bond strength,

A is the interfacial area $\left(\mathrm{mm}^{2}\right)$, and;

$P$ is the force $(N)$.

\section{Statistical Analysis}

One-way ANOVA and Tukey test were used to analyse the data using a statistical program (SPSS version 22, IBM, Chicago, IL) $(\mathrm{p}<0.05)$. Furthermore, mean \pm SD for surface treatment groups were presented through descriptive statistics.

\section{RESULTS}

\begin{tabular}{|c|c|c|c|c|c|}
\hline \multicolumn{7}{|c|}{ Descriptive Statistics } \\
\hline & N & Minimum & Maximum & Mean & S.D. \\
\hline Group 1 & 19 & 25.3700 & 216.2400 & 104.294211 & 50.0917033 \\
\hline Group 2 & 14 & 11.98 & 209.81 & 83.6457 & 53.56625 \\
\hline Group 3 & 19 & 44.0900 & 188.7700 & 124.342105 & 43.3786934 \\
\hline Group 4 & 18 & 5.2400 & 194.7600 & 94.141111 & 57.9162065 \\
\hline \multicolumn{7}{|c|}{ Table 1. Shear Bond Strength of Lithium Disilicate with } \\
Different Surface Treatments (Mean \pm SD) \\
\hline Note: SD = Standard Deviation
\end{tabular}

\begin{tabular}{|c|c|c|c|c|c|c|c|}
\hline \multicolumn{8}{|c|}{ ANOVA } \\
\hline & \multicolumn{3}{|c|}{ Sum of Squares } & df & Mean Square & $\mathbf{F}$ & Sig. \\
\hline \multicolumn{2}{|c|}{ Between Groups } & \multicolumn{2}{|c|}{15342.922} & 3 & 5114.307 & 1.947 & .031 \\
\hline \multicolumn{2}{|c|}{ Within Groups } & \multicolumn{2}{|c|}{173360.356} & 66 & \multirow[t]{2}{*}{2626.672} & & \\
\hline Total & & 188 & 03.279 & 69 & & & \\
\hline \multicolumn{8}{|c|}{ The one-way ANOVA: statistically significant at $p<0.05$} \\
\hline \multicolumn{8}{|c|}{ Tukey HSD } \\
\hline & & & \multicolumn{2}{|c|}{$\begin{array}{c}\text { Mean } \\
\text { Difference }\end{array}$} & \multicolumn{2}{|l|}{ Std. Error } & Sig \\
\hline \multirow{3}{*}{ Group 1} & & Group 2 & \multicolumn{2}{|c|}{6.68} & \multicolumn{2}{|l|}{1.891} & 0.003 \\
\hline & & Group 3 & \multicolumn{2}{|c|}{6.10} & \multicolumn{2}{|l|}{1.891} & 0.008 \\
\hline & & Group 4 & \multirow{2}{*}{\multicolumn{2}{|c|}{$\frac{12.12}{6.68}$}} & \multicolumn{2}{|l|}{1.891} & 0.000 \\
\hline \multirow{3}{*}{ Group 2} & & Group 1 & & & 4.891 & & 0.001 \\
\hline & & Group 3 & \multicolumn{2}{|c|}{6.65} & \multicolumn{2}{|l|}{1.891} & 0.000 \\
\hline & & Group 4 & \multicolumn{2}{|c|}{6.20} & \multicolumn{2}{|l|}{1.891} & 0.000 \\
\hline \multirow{3}{*}{ Group3 } & & Group 1 & \multirow{2}{*}{\multicolumn{2}{|c|}{$\begin{array}{c}8.14 \\
11.51\end{array}$}} & \multicolumn{2}{|l|}{1.891} & 0.001 \\
\hline & & Group 2 & & & 1.891 & & 0.003 \\
\hline & & Group 4 & \multicolumn{2}{|c|}{8.64} & 1.891 & & 0.000 \\
\hline
\end{tabular}

The highest mean was observed in Group 3 (124.34 \pm 43.47$)$ followed by Group $1(104.29 \pm 50.09)$, which is control group. In contrast, the lowest mean was observed in Group 2 (83.64 \pm 53.56$)$ and Group 4 (94.14 \pm 57.91$)$. Mean \pm standard deviation for shear bond strength of lithium disilicate with different surface treatments are presented in Table 1. Table 2 shows one-way ANOVA with post-hoc Tukey test for surface treatments. The results of ANOVA test have shown a 
significant difference between the surface treatment groups. The statistical power of the performed test is 0.031 at 0.05 significance level.

\section{DISCUSSION}

This study has evaluated and compared the tensile bonding strength after the ceramic surface was conditioned with different surface treatments. Traditionally, HF acid etching and primer is carried out separately as a two-step technique. Nevertheless, recently, both procedures are completed in a one-step conditioning system. The results of this study showed and compared between one-step conditioning system and the two-step conditioning system as part of this study. One-step conditioning system had lower adhesion strength values than the two-step conditioning system.

A study by Prochnow et al. ${ }^{20}$ also reported lower adhesion strength values with the one-step conditioning system. This is because of the weak acidic salt of hydrofluoric acid in the ammonium poly- fluoride (poly- $\mathrm{NH}_{4} \mathrm{HF}_{2}$ ) that the one-step conditioning system Monobond TM Etch and Prime.

The 5\% HF acid etch had been used in the two-step conditioning system, which creates low surface roughness. Siqueira et al. ${ }^{21}$ have also reported that partial dissolution of the glassy phase will be created, when ceramic surfaces were etched by using one step conditioning system. Poor resin cements flow into the micropores could be explained by surface roughness and mechanical interlocking. The low surface roughness adversely affects the mechanical interlocking with resin tags. ${ }^{22}$ Chemical bonding with a silane coupling agent requires adequate acid etching to exposed hydroxyl ions to facilitate good bonding strength. ${ }^{23}$

The specimens used in Group 3 showed a greater bond strength without curing of bonding agent that applied on tooth surface as compared to cure bonding agent of tooth surface in Group 1 (control group). This could be related to the intimate contact of enamel to light cure resin cement, consequently, to improve the overall bond strength. Tribst et al ${ }^{11}$ have presented both ceramics for HF-treated surfaces based on contact angles and wettability. The study has indicated that the exposure of chemical ligands and removal of the glass matrix is likewise in both tested ceramics. However, the surface wettability should be influenced by any overtreatment for increasing the number of pores. Tribst et al ${ }^{11}$ have further assessed and compared the ceramic-resin cement adhesion strength after undertaking one-step and two-step conditioning systems. Acid etching and primer application was independently carried out in the conventional two-step conditioning system. The study has found some differences between the resin composite and adhesive resin for some fundamental cement attributes, which include viscosity, mode of curing, tension, and wettability. Ramakrishnaiah et al ${ }^{8}$ have studied the influences of disinfectant, saliva, hydrogen peroxide contamination, and desensitizer to investigate adhesive-dentin bonding using a clinical try-in process. The findings obtained did not support the findings of the current study and indicated that the tensile bond strengths of both adhesive-ceramic interfaces and adhesive dentin are similar for all dentin surface situations.
Thereby, this study has rejected that there is no difference in tensile bonding strength between surface treatment protocols. The results have indicated that the silane aspect was not effective to optimize the ceramic resin bond before applying the universal adhesive. It is argued that pre-treated lithium disilicate along with silane should be used by clinicians before applying the universal adhesive. This claim has been corroborated by the findings of previous study that the implementation of silane improves microshear bond strength between composite resin and lithium disilicate from 4.10 MPa to $14.58 \mathrm{MPa}^{4,8}$ Furthermore, when silane was applied with etched HF to lithium disilicate, the microshear bond strength was improved from 14.04 MPa to $24.70 \mathrm{MPa} .^{8}$

The importance of topographic change is to maintain adhesive strength as better micromechanical bonding area is allowed through an increased number of widths and pores of various sizes. The wettability of the ceramic surface for the application of resin composites and silane-coupling agents is affected by this increase in surface roughness. ${ }^{20,21}$ The importance of adequately porous surface is observed for the durable cementation of both $\mathrm{Fd}$ and $\mathrm{Ld}$ ceramic indirect restorations. $^{24}$ Clean surfaces, the use of low-viscosity adhesives, cements, and suitable wetting are the other factors that contribute to the effectiveness of bonding between the materials. ${ }^{25}$

The results of the present study have recommended and indicated lower adhesion strength values when a one-step conditioning is used for ceramic surface as compared to the two-step conditioning system. Lower adhesion strength values with the one-step conditioning system were also reported by Prochnow et al..$^{20}$ The glassy phase in the ceramic is etched by using prime liquid and Monoband Etch for creating low surface roughness. Siqueira et $\mathrm{al}^{1}$ have indicated that the one-step conditioning system is used for the glassy phase when the ceramic surface was etched.

In bonding interfaces, adhesive systems tend to water sorption, which might absorb water from the ceramic structure, resulting in mechanical properties reduction and polymer plasticization, but might further offer water for the cleavage of siloxane bond.10 A significant reduction of bond strength at the interface is occurred over a time period, when a silanized interface is exposed to water due to the presence of hydrolytic cleavage of siloxane bonds in the siloxane. ${ }^{3}$ Therefore, all of the aforementioned concerns might contribute to a minimized bond strength investigated in all the groups of this study. Comparing the HF alone, the correlation between self-etching ceramic primer and HF did not indicate any significant modification in the modifying pattern of the lithium disilicate. In contrast, the less pronounced etching pattern is promoted through the self-etching ceramic primer, which leads to a partial rejection of the null hypothesis.

Similar findings have been reported by Siqueira et $\mathrm{al}^{1}$ that the association between the ceramic surface and ammonium polyfluoride achieves self-etching ceramic primer to produce the etching pattern. A partial dissolution of the glassy matrix is produced as compared to the production made by hydrofluoric acid where ammonium polyfluoride is comprised of milder acidity and acidic salt as compared to hydrofluoric acid. Furthermore, a partial dissolution is yet sufficient for the promotion of an adhesive interlocking with the ceramic 
surface. Therefore, the self-etching ceramic primer cannot be observed as a drawback to produce the less-pronounced etching pattern. It should be noted that any significant benefits cannot be brought due to a more-pronounced etching pattern with respect to its bond strength.

The smear layer and depleted fluoride ions were removed from the acid-etching procedure in the dentin surface, which allow a hybrid layer formation. The tubules are penetrated by resin monomers, which form resin tags with a jagged-like characteristic. In contrast, the etching procedure is neutralized by the deposition of fluoride ions on the dentin surface as well as inhibited a hybrid layer formation. ${ }^{26,27}$ These effects of fluoride deposition on the dentin surface might correspondingly interfere with the bonding process, which indicated that dentin bond strength might be decreased by fluoride-containing desensitized.

Several limitations in this study have been observed. Firstly, only one type of resin cement was used in this study. Secondly, the interpretation of the results was restricted due to the small number of specimens as a higher number of specimens might produce substantial differences with a smaller standard deviation. Thirdly, crystallographic analyses and surface roughness measurements were not examined in this study. Lastly, not curing the bonding agent of the total etch results a higher bond strength. Therefore, future studies are suggested to observe adhesive luting since modifications in wetting ability, mechanical properties and viscosity, and chemical compositions can further impact the adhesive properties of resin cement. Furthermore, both silanization and etching should be performed prior to bonding for lithium disilicate. It is suggested to use different resin cements to evaluate the same present hypothesis. Many available universal adhesives have diverse compositions, which can be used in different interactions with the surface pre-treatment method.

\section{CONCLUSIONS}

The use of HF etching and silane to lithium disilicate before applying a universal adhesive, improves resin-lithium disilicate bond strength. It is important to apply a regimen of $5 \% \mathrm{HF}$ at 20 seconds for reducing surface damage to the ceramic when using the universal adhesive along with silane. Similarly, the use of $9.5 \%$ HF can increase bond strength for 60 seconds if an additional step is not applied before adding a universal adhesive. Once self-etching achieves the chemical interaction and long-term strength than the conventional treatment, it can be treated as an alternative to conventional ceramic treatment. The self-etching system is important to maintain adhesive bonds to glass ceramics on the basis of positive outcomes obtained for the surface treatments as well as for the possible risks related with HF procedures.

\section{REFERENCES}

[1] Siqueira FS, Campos VS, Wendlinger M, et al. Effect of selfetching primer associated to hydrofluoric acid or silane on bonding to lithium disilicate. Brazilian Dental Journal 2019;30(2):171-8.

[2] Swank H, Bailey C, Motyka N, et al. Bond strength of resin cement to ceramic with new simplified primers and pretreatment solutions. 59th Medical Wing (AETC), JBSALackland, Texas, United States: 18 May 2016.

[3] Sofan E, Sofan A, Palaia G, et al. Classification review of dental adhesive systems: from the IV generation to the universal type. Annal Di Stomatologia (Roma) 2017;8(1):1-17.

[4] Kalavacharla VK, Lawson NC, Ramp LC, et al. Influence of etching protocol and silane treatment with a universal adhesive on lithium disilicate bond strength. Operative Dentistry 2015;40(4):372-8.

[5] Bajraktarova-Valjakova E, Korunoska-Stevkovska V, Kapusevska B, et al. Contemporary dental ceramic materials, a review: chemical composition, physical and mechanical properties, indications for use. Open Access Macedonian Journal of Medical Sciences 2018;6(9):174255.

[6] Yilmaz-Savas T, Demir N, Ozturk AN, et al. Effect of different surface treatments on the bond strength of lithium disilicate ceramic to the zirconia core. Photomedicine and Laser Surgery 2016;34(6):236-43.

[7] Lapinska B, Rogowski J, Nowak J, et al. Effect of surface cleaning regimen on glass ceramic bond strength. Molecules 2019;24(3):389.

[8] Ramakrishnaiah R, Alkheraif A, Divakar D, et al. The effect of hydrofluoric acid etching duration on the surface micromorphology, roughness and wettability of dental ceramics. International Journal of Molecular Sciences 2016;17(6):822.

[9] Saker S, Alnazzawi A, Özcan M. Adhesive strength of selfadhesive resins to lithium disilicate ceramic and dentin: effect of dentin chelating agents. Odontology 2016;104(1):53-9.

[10] Sundfeld D, Palialol AR, Fugolin AP, et al. The effect of hydrofluoric acid and resin cement formulation on the bond strength to lithium disilicate ceramic. Brazilian Oral Research 2018;32:e43.

[11] Tribst JP, Anami LC, Özcan M, et al. Self-etching primers vs acid conditioning: impact on bond strength between ceramics and resin cement. Operative Dentistry 2018;43(4):372-9.

[12] Ataol AS, Ergun G. Repair bond strength of resin composite to bilayer dental ceramics. The Journal of Advanced Prosthodontics 2018;10(2):101-12.

[13] AlRabiah M, Labban N, Levon JA, et al. Bond strength and durability of universal adhesive agents with lithium disilicate ceramics: a shear bond strength study. Journal of Adhesion Science and Technology 2018;32(6):580-9.

[14] Gundogdu M, Aladag LI. Effect of adhesive resin cements on bond strength of ceramic core materials to dentin. Nigerian Journal of Clinical Practice 2018;21(3):367-74.

[15] Zortuk M, Kilic K, Gurbulak AG, et al. Tensile bond strength of a lithium-disilicate pressed glass ceramic to dentin of different surface treatments. Dental Materials Journal 2010;29(4):418-24. 
[16] Mallikarjuna DM, Kumar SGB, Shetty S, et al. Comparative evaluation of lithium disilicate ceramic surface and bond strength to dentin surface after treatment with hydrofluoric acid and acidulated phosphate fluoride gel: an in vitro study. Indian Journal of Dental Research 2018;29(6):794-8.

[17] Lyann SK, Takagaki T, Nikaido T, et al. Effect of different surface treatments on the tensile bond strength to lithium disilicate glass ceramics. Journal of Adhesive Dentistry 2018;20(3):261-8.

[18] Alnassar T, Vohra F, Abualsaud H, et al. Efficacy of novel cleansing agent for the decontamination of lithium disilicate ceramics: a shear bond strength study. Journal of Adhesion Science and Technology 2017;31(2):202-10.

[19] Abduljabbar T, AlQahtani MA, AlJeaidi Z, et al. Influence of silane and heated silane on the bond strength of lithium disilicate ceramics - an in vitro study. Pakistan Journal of Medical Sciences 2016;32(3):550-4.

[20] Prochnow C, Prado M, Marchionatti AME, et al. One-step surface conditioning of two glass-ceramics: resin bond durability. Dental Materials 2017;33:e65.

[21] Siqueira FS, Alessi RS, Cardenas AF, et al. New singlebottle ceramic primer: 6-month case report and laboratory performance. The journal of contemporary dental practice. J Contemo Dent Pract 2016;17(12):10339.
[22] Ho GW, Matinlinna JP. Insights on ceramics as dental materials. Part II: chemical surface treatments. Silicon 2011;3:117.

[23] Matinlinna JP, Vallittu PK. Silane based concepts on bonding resin composite to metals. J Contemp Dent Pract 2007;8(2):1-8.

[24] Sattabanasuk V, Charnchairerk P, Punsukumtana L, et al. Effects of mechanical and chemical surface treatments on the resin-glass ceramic adhesion properties. Journal of Investigative and Clinical Dentistry 2017;8(3):e12220.

[25] Souza RO, Castilho AA, Fernandes VV, et al. Durability of microtensile bond to nonetched and etched feldspar ceramic: self-adhesive resin cements vs conventional resin. J Adhes Dent 2011;13(2):155-62.

[26] Marocho SM, Ozcan M, Amaral R, et al. Effect of resin cement type on the microtensile bond strength to lithium disilicate ceramic and dentin using different test assemblies. J Adhes Dent 2013;15(4):361-8.

[27] Koin PJ, Kilislioglu A, Zhou M, et al. Analysis of the degradation of a model dental composite. Journal of Dental Research 2008;87(7):661-5. 